\begin{tabular}{|c|l|}
\hline Title & A leukemic leukemia cutis in a patient with Philadel phia chromosome positive biphenoty pic leukemia. \\
\hline Author(s) & $\begin{array}{l}\text { Onozawa, Masahiro; Hashino, Satoshi; Kanamori, Hiroe; Izumiyama, Koh; Y onezumi, Masakatsu; Chiba, Koji; Kondo, } \\
\text { Takeshi; Fukuhara, Takashi; Tanaka, Junji; Imamura, Masahi ro; A saka, Masahiro }\end{array}$ \\
\hline Citation & International Journal of Hematology, 80(3), 278-280 \\
\hline Issue Date & 200410 \\
\hline Doc URL & http:/hdl.handle.net/2115/18871 \\
\hline Type & article \\
\hline File Information & IJH80-3.pdf \\
\hline
\end{tabular}

Instructions for use 


\title{
Aleukemic Leukemia Cutis in a Patient with Philadelphia Chromosome-Positive Biphenotypic Leukemia
}

\author{
Masahiro Onozawa, ${ }^{a}$ Satoshi Hashino, ${ }^{\text {a }}$ Hiroe Kanamori, ${ }^{a}$ Koh Izumiyama, ${ }^{a}$ Masakatsu Yonezumi, ${ }^{a}$ \\ Koji Chiba, ${ }^{a}$ Takeshi Kondo, ${ }^{a}$ Takashi Fukuhara, ${ }^{b}$ Junji Tanaka, ${ }^{c}$ Masahiro Imamura, ${ }^{\mathrm{c}}$ Masahiro Asaka ${ }^{\mathrm{a}}$ \\ Departments of ${ }^{a}$ Gastroenterology and Hematology and ${ }^{c}$ Hematology and Oncology, \\ Hokkaido University Graduate School of Medicine, Sapporo; ${ }^{b}$ Department of Internal Medicine, \\ Asahikawa City Hospital, Asahikawa, Japan
}

Received January 23, 2004; received in revised form June 18, 2004; accepted June 18, 2004

\begin{abstract}
Aleukemic leukemia cutis is a rare condition characterized by the invasion of leukemic blasts into the skin before their appearance in the peripheral blood. Leukemia cutis usually occurs in patients with myeloid leukemia, especially the myelomonocytic and monocytic types of acute myeloblastic leukemia. We describe the case of a 62-year-old woman with aleukemic leukemia cutis who developed Philadelphia-positive acute leukemia 1 month after skin involvement. Leukemic cells expressed both myeloid and B-cell lineage surface markers, and monoclonal rearrangement of the immunoglobulin heavy chain was detected by Southern blot analysis. This report is the first of a case of aleukemic leukemia cutis preceding Philadelphia-positive biphenotypic leukemia.
\end{abstract}

Int J Hematol. 2004;80:278-280. doi: 10.1532/IJH97.04010

(C)2004 The Japanese Society of Hematology

Key words: Aleukemic leukemia cutis; Philadelphia chromosome; Biphenotypic leukemia

\section{Introduction}

Aleukemic leukemia cutis (ALC) is a rare form of leukemia manifestation. A review of the literature showed that there have been 32 reported cases of ALC [1]. Leukemia subsequently developed in most of the reported cases, and most cases had a poor clinical course. Here we describe the first reported case of ALC caused by Philadelphia $(\mathrm{Ph})$ chromosome-positive biphenotypic leukemia. The existence of the $\mathrm{Ph}$ chromosome in our case may have contributed to the early development of acute leukemia and the aggressive clinical course.

\section{Case Report}

A 62-year-old woman developed multiple erythematous dermal papules on May 1, 2001, and went to the dermatology department of a local hospital on May 12. A complete

Correspondence and reprint requests: Masahiro Onozawa, MD, Department of Gastroenterology and Hematology, Hokkaido University Graduate School of Medicine, Kita 14, Nishi 5, Kita-ku, Sapporo 060-8638, Japan; 81-11-716-1161; fax: 81-11-706-7867

(e-mail: masahiro.onozawa@nifty.ne.jp). blood cell count showed the following values: hemoglobin, $13.5 \mathrm{~g} / \mathrm{dL}$; hematocrit, $40.7 \%$; platelets, $211 \times 10^{9} / \mathrm{L}$; and white blood cells, $7.9 \times 10^{9} / \mathrm{L}$, with $51 \%$ neutrophils, $40 \%$ lymphocytes, $7 \%$ monocytes, and $2 \%$ basophils. The skin lesions did not respond to conventional ointment therapy, including steroid therapy. A skin biopsy obtained from a papule on May 22 showed a dense infiltration of mononuclear cells in the dermis and subcutaneous fat tissue. Biochemistry profiles were within normal limits except for the elevation of the lactate dehydrogenase level to $747 \mathrm{IU} / \mathrm{L}$ (normal range, 220-430 IU/L). The bone marrow was not investigated at that time. Initially, cutaneous lymphoma of the skin was suspected on the basis of the biopsy results. The patient was referred to the Dermatology Department of Hokkaido University Hospital on June 5, when leukemic blasts appeared in peripheral blood. The patient was referred to our department with a suspicion of overt leukemia.

A physical examination revealed a widespread eruption comprising erythematous dermal papules located on the trunk, face, and extremities (Figure 1). There was no lymphadenopathy or hepatosplenomegaly.

A complete blood cell count showed the following values: hemoglobin, $9.8 \mathrm{~g} / \mathrm{dL}$; hematocrit, $29.2 \%$; platelets, $99 \times$ $10^{9} / \mathrm{L}$; and white blood cells, $10.7 \times 10^{9} / \mathrm{L}$, with $20 \%$ neutrophils, $19 \%$ lymphocytes, $3 \%$ monocytes, $2 \%$ basophils, $2 \%$ metamyelocytes, $2 \%$ myelocytes, and $53 \%$ blast cells. 


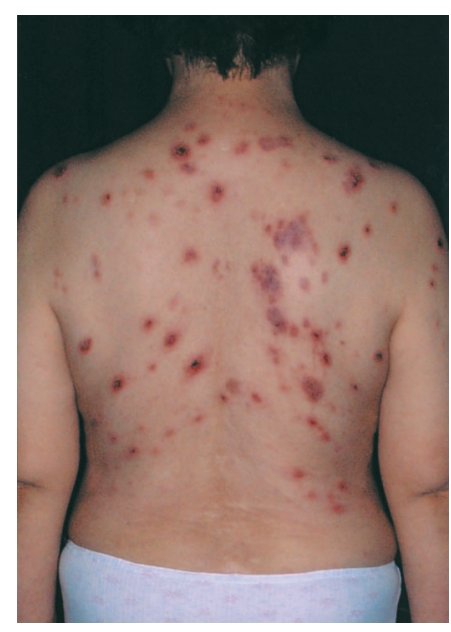

Figure 1. Widespread erythematous dermal papules. Multiple papules on the back measuring $10 \mathrm{~mm}$ to $30 \mathrm{~mm}$ in diameter.

The biochemistry profile revealed an elevation of the lactate dehydrogenase level to $637 \mathrm{IU} / \mathrm{L}$ (normal range, 200$460 \mathrm{IU} / \mathrm{L}$ ). A bone marrow aspirate on June 6 showed hypercellularity with $89 \%$ blast cells. The blast cells expressed B-cell and myeloid antigens, including CD10, CD19, CD13, CD34, and CD68, and showed a positive reaction to myeloperoxidase. Double-staining analysis revealed that the blasts comprised a single population and expressed both CD13 and CD19 (Figure 2). On the other hand, the blasts were negative for CD3, CD4, CD20, CD33, and CD56. A monoclonal rearrangement of the immunoglobulin heavy chain was detected by a Southern blot analysis using bone marrow cells. The patient's diagnosis was biphenotypic leukemia according to the scoring system of Catovski et al [2]. A cytogenetic examination demonstrated a mixed karyotypic pattern consisting of 46,XX,t(9;22)(q34;q11)[18/20], 46,XX,idem, der(19)t(1;19)(q23;p13)[1/20], and 46,XX[1/20]. The results of a reverse transcriptase-polymerase chain reaction analysis were positive for both major and minor $B C R /$

\begin{tabular}{lc}
\multicolumn{2}{c}{ Single-staining scatter } \\
CD2 & $22.0 \%$ \\
CD3 & $10.4 \%$ \\
CD4 & $11.2 \%$ \\
CD7 & $34.7 \%$ \\
CD8 & $7.82 \%$
\end{tabular}

\begin{tabular}{ll} 
CD10 & $66.2 \%$ \\
\hline CD11 & $17.7 \%$ \\
CD13 & $61.2 \%$ \\
\hline CD16 & $12.8 \%$ \\
CD19 & $71.6 \%$ \\
\hline
\end{tabular}

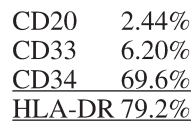

Double-staining scatter

\begin{tabular}{ll} 
CD19(+)13(+) & $57.8 \%$ \\
\hline CD19(+)13(-) & $13.4 \%$ \\
CD19(-)13(+) & $2.18 \%$ \\
CD19(-)13(-) & $26.6 \%$
\end{tabular}

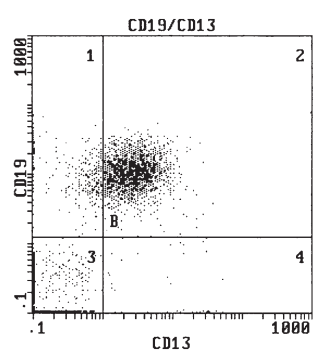

Figure 2. Flow cytometric analysis of bone marrow blasts.
$A B L$ transcripts. A diagnosis of $\mathrm{Ph}$ chromosome-positive biphenotypic leukemia was finally made.

A skin biopsy obtained from a papule on the patient's back on June 5 showed a dense infiltration of mononuclear cells in the dermis and subcutaneous fat tissue (Figure 3). Immunohistochemical staining revealed that the blast cells were positive for MT-1 (CD43), CD68, and myeloperoxidase and negative for CD3, CD20, and CD56. The cells infiltrating the skin also expressed multilineage surface markers and were compatible with leukemia cutis caused by biphenotypic leukemia. ALC preceding Ph chromosome-positive biphenotypic leukemia was finally diagnosed.

The patient underwent induction chemotherapy consisting of cytarabine and idarubicin. Although the skin lesions improved dramatically, this treatment resulted in only partial regression of the leukemic blasts in the bone marrow. Despite 2 additional courses of reinduction chemotherapy (1 course with cyclophosphamide, daunorubicin, vincristine, and prednisolone and the other course with cyclophosphamide, prednisolone, mitoxantrone, and cytarabine), the patient died of sepsis 3 months after the diagnosis of overt leukemia and 4 months after the initial presentation of leukemia cutis. An autopsy was not permitted.

\section{Discussion}

Cutaneous lesions in leukemia are divided into 2 groups: leukemid or nonspecific lesions, in which inflammatory lesions contain no leukemic cells, and leukemia cutis or specific lesions, in which leukemic cells invade the skin [3]. ALC is a rare condition in which leukemia cutis precedes the diagnosis of systemic leukemia [4]. Chang et al presented a review of 31 cases of ALC. Of 18 cases in which a leukemic cell lineage was documented, 17 cases were M4/5 by the French-American-British classification, and 1 case was M2. A few other cases of ALC caused by lymphoblastic leukemia have been reported [5,6]; however, there has been no case report of ALC caused by biphenotypic leukemia.

ALC may sometimes be misdiagnosed as cutaneous lymphoma or undifferentiated carcinoma. In most cases, ALC is diagnosed after bone marrow infiltration has been found, and then antileukemic treatment is initiated, as in our case.

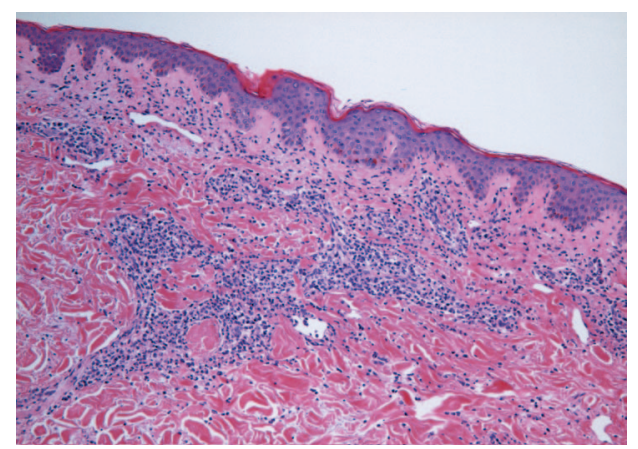

Figure 3. Dermal infiltrate of leukemic blasts. Photomicrograph of skin biopsy specimen shows diffuse dermal infiltration with atypical cells (hematoxylin and eosin, original magnification $\times 10$ ). 
There is no consensus regarding the treatment modality for ALC and the timing of initial treatment. Although treatment regimens with combination chemotherapy and/or electronbeam radiation therapy have been reported, the prognosis is always poor [7]. Only 2 patients have been treated before progression to acute leukemia, and they survived without recurrence for 16 months and 30 months $[1,8]$. The times from cutaneous onset to systemic expansion of leukemic cells varied from 1 month to 18 months [5,9]. These cases of ALC raise questions about the origin of neoplastic clones and their biological behavior. It is not clear whether malignant cells originate in the bone marrow and subsequently migrate to the skin or malignant cells originate in skin and then migrate to the bone marrow.

The $\mathrm{Ph}$ chromosome is found in most cases of chronic myelogenous leukemia, in approximately $20 \%$ of adult and $5 \%$ of pediatric cases of acute lymphoblastic leukemia (ALL), and in approximately $2 \%$ of acute myeloblastic leukemia (AML) cases [10]. Among ALL and AML cases, the presence of the $\mathrm{Ph}$ chromosome is a predictor of a high incidence of induction failure and relapse after chemotherapy, which are related to a poor prognosis [10]. Biphenotypic leukemia is a relatively rare leukemia, identified in $5 \%$ to $10 \%$ of acute leukemias. On the other hand, biphenotypic features are common $(47 \%)$ in patients with $\mathrm{Ph}$ chromosome-positive acute leukemia [11,12]. Among biphenotypic leukemia cases, the presence of the Ph chromosome is also a poor prognostic factor [13]. The existence of the $\mathrm{Ph}$ chromosome may have resulted in the aggressive clinical course observed in our patient. Although, imatinib mesylate might have been temporally effective in this situation [14,15], it was not yet commercially available in Japan at that time.

In conclusion, leukemia cutis may precede systemic involvement of $\mathrm{Ph}$ chromosome-positive biphenotypic leukemia. In spite of intensive combination chemotherapy, our patient had an aggressive clinical course without achievement of complete remission.

\section{Acknowledgments}

We thank Madoka Kato and Tetsuri Matsumura (Department of Dermatology, Hokkaido University Hospital) for the dermatologic diagnosis and advice on therapy.

\section{References}

1. Chang H, Shin LY, Kuo TT. Primary aleukemic myeloid leukemia cutis treated successfully with combination chemotherapy: report of a case and review of the literature. Ann Hematol. 2003;82: 435-439.

2. Catovsky D, Matutes E, Buccheri V, et al. A classification of acute leukemia for the 1990s. Ann Hematol. 1991;62:15-21.

3. Stawiski MA. Skin manifestations of leukemias and lymphomas. Cutis. 1978;21:814-818.

4. Yoder FW, Schuen RL. Aleukemic leukemia cutis. Arch Dermatol. 1976;112:367-369.

5. Taniguchi S, Hamada T, Kutsuna H, Ishii M. Lymphocytic aleukemic leukemia cutis. J Am Acad Dermatol. 1996;35:849-850.

6. Zengin N, Kars A, Ozisik Y, Canpinar H, Turker A, Ruacan S. Aleukemic leukemia cutis in a patient with acute lymphoblastic leukemia. J Am Acad Dermatol. 1998;38:620-621.

7. Ohno S, Yokoo T, Ohta M, et al. Aleukemic leukemia cutis. $J$ Am Acad Dermatol. 1990;22:374-377.

8. Longacre TA, Smoller BR. Leukemia cutis: analysis of 50 biopsyproven cases with an emphasis on occurrence in myelodysplastic syndromes. Am J Clin Pathol. 1993;100:276-284.

9. Barzilai A, Lyakhovitsky A, Goldberg I, Meytes D, Trau H. Aleukemic monocytic leukemia cutis. Cutis. 2002;69:301-304.

10. Kurzrock R, Kantarjian HM, Druker BJ, Talpaz M. Philadelphia chromosome-positive leukemias: from basic mechanisms to molecular therapeutics. Ann Intern Med. 2003;138:819-830.

11. Carbonell F, Swansbury J, Min T, et al. Cytogenetic findings in acute biphenotypic leukemia. Leukemia. 1996;10:1283-1287.

12. Matsuno Y, Drexler HG, Takeushi M, Orita K. A novel biphenotypic B-cell precursor leukemia cell line (NALM-29) carrying $\mathrm{t}(9 ; 22)(\mathrm{q} 34 ; \mathrm{q} 11)$ established from a patient with acute leukemia. Leukemia Res. 1999;23:731-740.

13. Legrand O, Perrot JY, Simonin G, et al. Adult biphenotypic acute leukaemia: an entity with poor prognosis which is related to unfavourable cytogenetics and P-glycoprotein over-expression. Brit J Haematol. 1998;100:147-155.

14. Inami M, Inokuchi K, Nakayama K, Tamura H, Shimada T, Dan K. Simultaneous novel $B C R-A B L$ gene mutation and increased expression of $B C R-A B L$ mRNA caused clinical resistance to STI571 in double-Ph-positive acute biphenotypic leukemia. Int $J$ Hematol. 2003;78:173-175.

15. Ottmann OG, Wassmann B. Imatinib in the treatment of Philadelphia chromosome-positive acute lymphoblastic leukaemia: current status and evolving concepts. Best Pract Res Clin Haematol. 2002; 15:757-769. 